\title{
Nonlinear wave interactions of kinetic sound waves
}

\author{
G. Brodin ${ }^{1}$ and L. Stenflo ${ }^{2}$ \\ ${ }^{1}$ Department of Physics, Umeå University, 90187 Umeå, Sweden \\ ${ }^{2}$ Department of Physics, Linköping University, 58183 Linköping, Sweden
}

Correspondence to: G. Brodin (gert.brodin @ physics.umu.se)

Received: 20 May 2015 - Revised: 5 July 2015 - Accepted: 29 July 2015 - Published: 14 August 2015

\begin{abstract}
We reconsider the nonlinear resonant interaction between three electrostatic waves in a magnetized plasma. The general coupling coefficients derived from kinetic theory are reduced here to the low-frequency limit. The main contribution to the coupling coefficient we find in this way agrees with the coefficient recently presented in Annales Geophysicae. But we also deduce another contribution which sometimes can be important, and which qualitatively agrees with that of an even more recent paper. We have thus demonstrated how results derived from fluid theory can be improved and generalized by means of kinetic theory. Possible extensions of our results are outlined.
\end{abstract}

Keywords. Magnetospheric physics (solar windmagnetosphere interactions)

\section{Introduction}

The nonlinear interaction between three waves in the lowfrequency range (i.e. below the ion-cyclotron frequency $\omega_{\mathrm{ci}}$ ) was studied in a recent paper (Lyubchyk and Voitenko, 2014) by means of a two-fluid plasma model. Such wave interactions are of basic interest in investigations of the solar corona and the solar wind, as well as in the Earth's magnetosphere and ionosphere, and the corresponding nonlinear phenomena (Shukla, 1999; Eliasson and Shukla, 2009) have also been observed by spacecrafts (Briand, 2009). It should be noted here that the space-frame frequencies measured in the solar wind plasma are strongly Doppler-shifted, and that the plasma rest-frame frequencies can be significantly lower than $\omega_{\mathrm{ci}}$. Lyubchyk and Voitenko (2014) have studied the nonlinear interaction of these waves in the electrostatic limit and outlined, with much physical insight, the decay processes as well as possible applications.

\section{The low-frequency electrostatic coupling coefficient}

In the present paper we are going to reconsider the way to deduce the results for nonlinear electrostatic wave interaction. Accordingly, we first remind the reader that it is possible to write the coupled equations for three waves satisfying matching conditions $\omega_{3}=\omega_{1}+\omega_{2}$ and $\boldsymbol{k}_{3}=\boldsymbol{k}_{1}+\boldsymbol{k}_{2}$ as in

$$
\frac{\mathrm{d} W_{1,2}}{\mathrm{~d} t}=-2 \omega_{1,2} \operatorname{Im} V
$$

and

$\frac{\mathrm{d} W_{3}}{\mathrm{~d} t}=2 \omega_{3} \operatorname{Im} V$

where $W=\varepsilon_{0} \mathbf{E}^{*} \cdot(1 / \omega) \partial\left(\omega^{2} \varepsilon\right) . \mathbf{E}$ is the wave energy, $\varepsilon$ is the usual textbook dielectric tensor (Swanson, 1989), and $\operatorname{Im} V$ stands for the imaginary part of $V$ (Stenflo, 1994; Brodin and Stenflo, 1990), where

$$
\begin{aligned}
V & =\sum_{\mathrm{s}} m \int \mathrm{d} \boldsymbol{v} F_{0}(\boldsymbol{v}) \sum_{\substack{p_{1}+p_{2}=p_{3} \\
p_{j}=0, \pm 1, \pm 2, \ldots}} I_{1}^{p_{1}} I_{2}^{p_{2}} I_{3}^{-p_{3}} \\
& \cdot\left[\frac{\boldsymbol{k}_{1} \cdot \boldsymbol{u}_{1 p_{1}}}{\omega_{1 d}} \boldsymbol{u}_{2 p_{2}} \cdot \boldsymbol{u}_{3 p_{3}}^{*}+\frac{\boldsymbol{k}_{2} \cdot \boldsymbol{u}_{2 p_{2}}}{\omega_{2 d}} \boldsymbol{u}_{1 p_{1}} \cdot \boldsymbol{u}_{3 p_{3}}^{*}\right. \\
+ & \left.\frac{\boldsymbol{k}_{3} \cdot \boldsymbol{u}_{3 p_{3}}^{*}}{\omega_{3 d}} \boldsymbol{u}_{1 p_{1}} \cdot \boldsymbol{u}_{2 p_{2}}-\frac{i \omega_{\mathrm{c}}}{\omega_{3 d}}\left(\frac{k_{2 z}}{\omega_{2 d}}-\frac{k_{1 z}}{\omega_{1 d}}\right) \boldsymbol{u}_{3 p_{3}}^{*} \cdot\left(\boldsymbol{u}_{1 p_{1}} \times \boldsymbol{u}_{2 p_{2}}\right)\right],
\end{aligned}
$$

where $F_{0}$ is the unperturbed distribution function, $\omega_{j d}=$ $\omega_{j}-k_{j z} v_{z}-p_{j} \omega_{\mathrm{c}}, I_{j}\left(=\exp \left(i \theta_{j}\right)\right)=\left(k_{j_{x}}+i k_{j y}\right) / k_{j \perp}, \omega_{\mathrm{c}}=$ $q B_{0} / m$ is the cyclotron frequency, $q / m$ the charge to mass ratio, and $\mathbf{B}_{0}=B_{0} \hat{z}$ is the external magnetic field. For notational convenience we have omitted the index " $\mathrm{s}$ " denoting particle species on all quantities. The general velocity vector $\boldsymbol{u}_{j p_{j}}$ has been presented previously by Stenflo (1994) and 
Brodin and Stenflo (2012). In the electrostatic limit it is

$$
\begin{aligned}
\boldsymbol{u}_{j p_{j}}= & \frac{q \Phi_{j}}{m \omega_{j d}\left(1-\omega_{\mathrm{c}}^{2} / \omega_{j d}^{2}\right)} \\
& \cdot\left(\boldsymbol{k}_{j}-\frac{i \omega_{\mathrm{c}}}{\omega_{j d}} \widehat{z} \times \boldsymbol{k}_{j}-\frac{\omega_{\mathrm{c}}^{2}}{\omega_{j d}^{2}} k_{j z} \widehat{z}\right) J_{p_{j}},
\end{aligned}
$$

where $\mathbf{E}_{j}=-i \boldsymbol{k}_{j} \Phi_{j}$ is the electric field amplitude of wave $j$ and $J_{p_{j}}$ is the Bessel function of order $p$ with argument $k_{j \perp} v_{\perp} / \omega_{\mathrm{c}}$. Furthermore, the wave energy density can be written $W_{j}=\omega_{j} \varepsilon_{0} k_{j}^{2}\left|\Phi_{j}\right|^{2} \partial\left(\varepsilon\left(\omega_{j}, \boldsymbol{k}_{j}\right)\right) / \partial \omega_{j}$, where $\varepsilon\left(\omega_{j}, \boldsymbol{k}_{j}\right)$ is the scalar dielectric function in the electrostatic limit, described by the well known formula (cf. Hasegawa, 1975; Swanson, 1989; Stenflo, 1994):

$\varepsilon\left(\omega_{j}, \boldsymbol{k}_{j}\right)=1+\sum_{s, p} \frac{q^{2}}{m \varepsilon_{0} k_{j}^{2}} \int \frac{\mathrm{d} \boldsymbol{v}}{\omega_{j d}}\left(\frac{p \omega_{\mathrm{c}}}{v_{\perp}} \frac{\partial F_{0}}{\partial v_{\perp}}+k_{j z} \frac{\partial F_{0}}{\partial v_{z}}\right) J_{p}^{2}$.

The coupling coefficient $V$ determines the growth rate for parametric instabilities. When wave 3 constitutes the pump wave, the growth rate $\gamma$ well above threshold for decay into waves 1 and 2 is given by

$\gamma^{2}=\frac{\omega_{1} \omega_{2}|V|^{2}}{W_{1} W_{2}}$.

Nonlinear wave phenomena involving electrostatic highfrequency waves have previously been studied by e.g. Yinhua et al. (1999). Here we will focus on the opposite regime with waves with frequencies well below the ion-cyclotron frequency $\omega_{\text {ci }}$. Waves in this regime are so-called kinetic sound waves (KSWs, see e.g. Lyubchyk and Voitenko, 2014 or Zhao et al., 2014b). Evaluating the electrostatic dispersion relation $\varepsilon(\omega, \boldsymbol{k})=0$ for a two-component plasma (electrons and ions) in the low-frequency limit we obtain

$$
1=-\frac{\omega_{\mathrm{pe}}^{2}}{k^{2} v_{\mathrm{te}}^{2}}+\omega_{\mathrm{pi}}^{2}\left[\frac{k_{z}^{2}}{k^{2}} \int \frac{G_{0}\left(v_{z}\right) \mathrm{d} v_{z}}{\left(\omega-k_{z} v_{z}\right)^{2}}+\frac{G_{1}}{k^{2}}\right],
$$

where $v_{\mathrm{te}}^{2}=1 /\left\langle v_{\mathrm{ze}}^{2}\right\rangle^{-1}$ and $\langle\ldots\rangle$ denotes averaging over the unperturbed distribution function. Here $G_{0}\left(v_{z}\right)$ is the ion distribution function renormalized according to

$G_{0}\left(v_{z}\right)=\frac{\int J_{0}^{2}\left(k_{\perp} v_{\perp} / \omega_{\mathrm{ci}}\right) F_{0}(\boldsymbol{v}) v_{\perp} \mathrm{d} v_{\perp}}{\int F_{0}(\boldsymbol{v}) v_{\perp} \mathrm{d} v_{\perp} \mathrm{d} v_{z}}$.

Furthermore

$G_{1}=\frac{\int 2 J_{1}^{2}\left(k_{\perp} v_{\perp} / \omega_{\mathrm{ci}}\right)\left(\partial F_{0}(\boldsymbol{v}) / \partial v_{\perp}\right) \mathrm{d} v_{\perp} \mathrm{d} v_{z}}{\int F_{0}(\boldsymbol{v}) v_{\perp} \mathrm{d} v_{\perp} \mathrm{d} v_{z}}$.

Under suitable approximations, the dispersion relation (see Eq. 7) agrees with the fluid approximation for KSWs (Lyubchyk and Voitenko, 2014):

$\omega^{2}=\frac{k_{z}^{2} c_{\mathrm{s}}^{2}}{1+k_{\perp}^{2} v_{\mathrm{ti}}^{2} / \omega_{\mathrm{ci}}^{2}}$, where $c_{\mathrm{s}}^{2}=k_{\mathrm{B}}\left(T_{e}+T_{i}\right) / m_{i}, v_{\mathrm{ti}}^{2}=k_{\mathrm{B}} T_{i} / m_{i}$ and $k_{\mathrm{B}}$ is the Boltzmann constant. To get this agreement we should drop the left hand side of Eq. (7) (this quasi-neutral approximation applies for $\left.\omega_{\mathrm{pi}}^{2} / \omega_{\mathrm{ce}}^{2} \gg 1\right)$, expand the Bessel functions keeping terms up to $k_{\perp}^{2} v_{\perp}^{2} / \omega_{\mathrm{ci}}^{2}$, and let $\left(\omega-k_{z} v_{z}\right)^{2} \rightarrow \omega^{2}-k_{z}^{2} v_{\mathrm{ti}}^{2}$ in the denominator of the integral over $v_{z}$ (which is a reasonable approximation if the phase velocity is larger than the ion thermal velocity, such that ion Landau damping is small). To perform this treatment consistently, we must also consider $\omega^{2} \simeq k_{z}^{2} c_{\mathrm{s}}^{2}$ as a valid first order approximation. As $\omega / k_{z}$ is of the order of $c_{\mathrm{s}}$, we assume here that the ion temperature is smaller than the electron temperature, in order to avoid large Landau damping of the interacting waves. We note that in general there is also a high-frequency branch of magnetized ion acoustic waves with frequencies above the ion-cyclotron frequency. That mode is not included in our treatment, however. From now on we are therefore concerned with three waves that fulfill Eq. (7), and where the approximation (Eq. 10) applies at least qualitatively.

We next evaluate the coupling coefficient $V$ in the same limit $\omega \ll \omega_{\mathrm{ci}}$. We then note that $V$ reduces to the comparatively very simple coefficient

$V_{\mathrm{lf}}=C \Phi_{1} \Phi_{2} \Phi_{3}^{*}$,

where

$C=C_{1}+C_{2}$,

with its two contributions given by

$$
\begin{aligned}
C_{1}= & \sum_{\mathrm{s}} \int \mathrm{d} v_{z} \frac{G_{\|}\left(v_{z}\right)}{m^{2}} \\
& \cdot \frac{i q^{3}}{\left(\omega_{1}-k_{1 z} v_{z}\right)\left(\omega_{2}-k_{2 z} v_{z}\right)\left(\omega_{3}-k_{3 z} v_{z}\right)} \\
& \cdot\left(\frac{k_{1 z}}{\omega_{1}-k_{1 z} v_{z}}+\frac{k_{2 z}}{\omega_{2}-k_{2 z} v_{z}}+\frac{k_{3 z}}{\omega_{3}-k_{3 z} v_{z}}\right)
\end{aligned}
$$

and

$$
\begin{aligned}
C_{2}= & \sum_{\mathrm{s}} \int \mathrm{d} v_{z} \frac{q^{3}}{m^{2} \omega_{\mathrm{c}}} \frac{G_{\|}\left(v_{z}\right)}{\left(\omega_{3}-k_{3 z} v_{z}\right)} \\
& \cdot\left(\frac{k_{1 z}}{\omega_{1}-k_{1 z} v_{z}}+\frac{k_{2 z}}{\omega_{2}-k_{2 z} v_{z}}+\frac{k_{3 z}}{\omega_{3}-k_{3 z} v_{z}}\right) \\
& \cdot\left(\frac{k_{2 z}}{\omega_{2}-k_{2 z} v_{z}}-\frac{k_{1 z}}{\omega_{1}-k_{1 z} v_{z}}\right) \frac{\left(\boldsymbol{k}_{1 \perp} \times \boldsymbol{k}_{2 \perp}\right)_{z}}{k_{1 z} k_{2 z} k_{3 z}}
\end{aligned}
$$

where $G_{\|}\left(v_{z}\right)=2 \pi \int_{0}^{\infty} J_{01} J_{02} J_{03} F_{0}(\boldsymbol{v}) v_{\perp} \mathrm{d} v_{\perp}$ and $J_{0 j}=$ $J_{0}\left(k_{j \perp} v_{\perp} / \omega_{\mathrm{c}}\right)$. Here we consider for simplicity only unperturbed distribution functions which have separable velocity dependences. The term $C_{1}$ is due here to the so-called scalar nonlinearity, whereas $C_{2}$ is due to the vector nonlinearity (see Zhao et al., 2015). This follows from Eq. (3) where the first three terms together constitute the scalar nonlinearity, whereas the fourth term corresponds to the vector nonlinearity. 
Let us first focus on the term $C_{2}$. We note that provided that Eq. (10) is fulfilled at least qualitatively, the electron contribution to $C_{2}$ is negligible as compared to the ion contribution. Provided the electron temperature is larger than the ion temperature, and finite Larmor radius effects are relatively small, we can simplify $C_{2}$ by letting $\omega_{j}-k_{j z} v_{z} \rightarrow \omega_{j}$ as well as $J_{0 j} \rightarrow 1$ in Eq. (14). If such fluid-type of approximations are made, $C_{2}$ coincides with the expression for the coupling coefficient presented in Lyubchyk and Voitenko (2014), used to describe the parametric excitation of KSW : s. However, our general expression also contains a term $C_{1}$ that cannot be neglected in general. We note that in $C_{1}$, both the electron and ion contributions must typically be kept, at least if the electron and ion temperatures are of the same order. Furthermore, if we only use an expansion in $\omega / \omega_{\mathrm{ci}}$, which applies if the angles of propagation obey $k_{j z} \sim k_{j \perp}, C_{1}$ is 1 order larger than $C_{2}$. This may suggest that $C_{1}$ is more important than $C_{2}$ in the (low-frequency) regime of consideration. However, this is not necessarily true. To clarify the situation we need to separate between the case where the pump wave (assumed to have index 3) fulfills $k_{3 z} \ll k_{3 \perp}$ (case 1) and the case with $k_{3 z} \sim k_{3 \perp}$ (case 2). We first consider case 1 . We note that the preferred decay channel has daughter waves that maximize the growth rate. As the growth rate is directly proportional to the coupling coefficient, and $C_{2}$ (but not $C_{1}$ ) increases with perpendicular wavenumber, we note that the maximum growth rate occurs for large perpendicular wavenumber fulfilling

$k_{1,2 \perp}^{2} \gg k_{1,2 z}^{2} \frac{\omega_{\mathrm{ci}}}{\omega_{1,2}}$,

in which case $C_{1}$ is small as compared to $C_{2}$. Thus for pump waves with $k_{3 z} \ll k_{3 \perp}$, the result of Lyubchyk and Voitenko (2014) is essentially confirmed. Nevertheless, we note the usefulness of kinetic theory presented here, as this theory is needed to describe the finite Larmor radius effects that saturates the growth of $C_{2}$ with $k_{1,2 \perp}^{2}$, as contained in the Bessel function dependence of $G_{\|}\left(v_{z}\right)$.

Next we consider case 2 . For moderate values of $k_{3 \perp} \sim$ $k_{3 z}$, the term $C_{2}$ still increases with perpendicular wave number of the daughter waves, but only linearly in $k_{1 \perp}$ as $\left(\boldsymbol{k}_{1 \perp} \times \boldsymbol{k}_{2 \perp}\right)_{z}=\left(\boldsymbol{k}_{1 \perp} \times \boldsymbol{k}_{3 \perp}\right)_{z}$. This means that we need

$k_{1,2 \perp} \gtrsim\left|k_{1.2 z}\right| \frac{\omega_{\mathrm{ci}}}{\omega_{1,2}}$

for $C_{2}$ to be of the same magnitude as $C_{1}$. Given the dispersion relation (Eq. 10), the condition (Eq. 16) means that we are in the kinetic regime. Thus both terms $C_{1}$ and $C_{2}$ must be kept, the Bessel functions cannot be expanded, and the substitution $\omega_{j}-k_{j z} v_{z} \rightarrow \omega_{j}$ should be avoided. Finally we note that the conditions (Eqs. 15 and 16) for large perpendicular wavenumbers can be forbidden due to the resonance conditions, in case the interacting waves are propagating in the same direction along the magnetic field. For counterpropagating waves (i.e. different signs of $k_{1 z}$ and $k_{2 z}$ ), however, these conditions can be satisfied. As a consequence, the maximum magnitude of $C_{2}$, which implies the strongest interaction, occurs for counterpropagating waves. This has previously been pointed out by Voitenko (1998). In addition, one can see that the factor $\left(k_{2 z} / \omega_{2 d}-k_{1 z} / \omega_{1 d}\right)$ in Eq. (3) also indicated this fact.

\section{Conclusions}

As described in some detail by Lyubchyk and Voitenko (2014), decays into electrostatic waves are of particular relevance for the solar wind plasma. However, it should be noted that other decay channels are also possible; see Brodin and Stenflo (1990), as well as Zhao et al. (2014a), wherein kinetic Alfvén waves are an important ingredient in the nonlinear interaction of the solar wind plasma. A relevant question is how the signature of the present process can be seen in spacecrafts' observations of the solar wind (Briand, 2009). The plasma rest-frame frequencies studied here will generally be Doppler-shifted by a term $k_{j} \cdot v_{\mathrm{s}}$, where $v_{\mathrm{s}}$ is the spacecraft velocity. Since the wavevectors of the interacting waves can differ both in directions and magnitude, the frequency shift will vary accordingly. In particular the frequency shifts of the daughter waves fulfilling the conditions (Eqs. 15 and 16) will be very large, unless the spacecraft propagates parallel to the magnetic field. The pump wave can be scattered both forwards and backwards, depending on the particular situation.

Finally, we stress that the present coupling coefficient (Eq. 12) which has been derived for a collisionless plasma, can be significantly changed when collisional effects are taken into account (Stenflo, 1971; Kuo et al., 1998; Bulgakov and Shramkova, 2007). This is however outside the scope of the present work, but has to be taken into account in future applications.

The topical editor C. Owen thanks B. Eliasson and one anonymous referee for help in evaluating this paper.

\section{References}

Briand, C.: Plasma waves above the ion cyclotron frequency in the solar wind: a review on observations, Nonlin. Processes Geophys., 16, 319-329, doi:10.5194/npg-16-319-2009, 2009.

Brodin, G. and Stenflo, L.: Coupling coefficients for ioncyclotron Alfvén waves, Contrib. Plasma Phys., 30, 413-419, doi:10.1002/ctpp.2150300308, 1990.

Brodin, G. and Stenflo, L.: Three-wave coupling coefficients for a magnetized plasma, Phys. Scripta, 85, 035504, doi:10.1088/0031-8949/85/03/035504, 2012.

Bulgakov, A. A. and Shramkova, O. V.: Nonlinear interaction of waves in semiconductor plasma, J. Phys. D: Appl. Phys., 40, 5896, doi:10.1088/0022-3727/40/19/017, 2007.

Eliasson, B. and Shukla, P. K.: New developments in nonlinear plasma physics, Proceedings of the 2009 ICTP Summer Col- 
lege on plasma physics and International Symposium On Cutting Edge Plasma Physics, AIP conference proceedings, Trieste, Italy, 10-28 August 2009, 1188, 2009.

Hasegawa, A.: Plasma instabilities and nonlinear effects, SpringerVerlag, Berlin, Germany, 68-79, 1975.

Kuo, S. P., Huang, J., and Lee, M. C.: Parametric excitation of ion Bernstein waves by parallel propagating Langmuir waves in a collisional plasma, J. Atmos. Solar-Terr. Phys., 60, 121-128, doi:10.1016/S1364-6826(97)00073-4, 1998.

Lyubchyk, O. and Voitenko, Y.: Nonlocal nonlinear coupling of kinetic sound waves, Ann. Geophys., 32, 1407-1413, doi:10.5194/angeo-32-1407-2014, 2014.

Shukla, P. K.: Nonlinear plasma science, Phys. Scripta T, 82, 1-141, 1999.

Stenflo, L.: Nonlinear interaction of electrostatic waves in a plasma, Annalen der Physik, 7, 289-295, doi:10.1002/andp.19714820306, 1971.

Stenflo, L.: Resonant three-wave interactions in plasmas, Phys. Scripta T, 50, 15-19, doi:10.1088/0031-8949/1994/T50/002, 1994.
Swanson, D. G.: Waves in a hot magnetized plasma, in: Plasma waves, Chap 4.3.3, Academic Press, London, UK, 142-162, 1989.

Voitenko, Y. M.: Three-wave coupling and weak turbulence of kinetic Alfven waves, J. Plasma Phys., 60, 515-527, 1998.

Yinhua, C., Wei, L., and Yu, M. Y.: Nonlinear electrostatic waves in a magnetized plasma, Phys. Rev. E, 60, 3249-3252, doi:10.1103/PhysRevE.60.3249, 1999.

Zhao, J. S., Voitenko, Y. M., Wu, D. J., and De Keyser, J.: Nonlinear Generation of Kinetic-Scale Waves by MHD Alfvén Waves and Nonlocal Spectral Transport in the Solar Wind, Astrophys. J., 785, 139, doi:10.1088/0004-637X/785/2/139, 2014a.

Zhao, J. S., Voitenko, Y. M., Yu, M. Y., Lu, J. Y., and Wu, D. J.: Properties of Short-wavelength Oblique Alfven and Slow waves, Astrophys. J., 793, 107, doi:10.1088/0004-637X/793/2/107, 2014b.

Zhao, J. S., Voitenko, Y. M., De Keyser, J., and Wu, D. J.: Scalar and vector nonlinear decays of low-frequency Alfven waves, Astrophys. J., 799, 222, doi:10.1088/0004-637X/799/2/222, 2015. 